Tendências em Matemática Aplicada e Computacional, 3, No. 1 (2002), 111-119.

(C) Uma Publicação da Sociedade Brasileira de Matemática Aplicada e Computacional.

\title{
Funções e Novas Tecnologias
}

V. GIRALDO ${ }^{1}$, Departamento de Métodos Matemáticos, Instituto de Matemática, UFRJ, Avenida Brigadeiro Trompowski s/n, 21945-970 Rio de Janeiro, RJ, Brasil

L.M. CARVALHO ${ }^{2}$, Departamento de Matemática Aplicada, Instituto de Matemática e Estatística, UERJ, Rua São Francisco Xavier 524, 20550-013 Rio de Janeiro, RJ, Brasil.

Resumo. Apresentamos e analisamos algumas atividades para ensino de funções com recursos computacionais, direcionadas a alunos de graduação e professores de ensino médio. Procuramos estimular a conexão entre as três representações fundamentais de funções (analítica, gráfica e numérica) a partir da análise crítica de resultados muitas vezes inesperados fornecidos pela máquina.

\section{Introdução}

O nosso objetivo central é apresentar e analisar atividades computacionais que visem favorecer a construção das ligações entre as três principais formas de representação (analítica, gráfica e numérica) de uma função real de variável real.

Em [4], apresentamos um plano de investigação sobre o ensino-aprendizagem de funções em ambientes com novas tecnologias. Dando continuidade a essa pesquisa, avançamos aqui no sentido proposto por Sierpinska [6], quando fala de funções e suas representações:

A consciência das limitações de cada uma das representações e do fato que elas representam um único conceito geral são, com certeza, condições fundamentais para a compreensão das funções.

Em relação às conexões entre as três principais representações de funções, ao observarmos os procedimentos de alunos recém-saídos do nível médio, constatamos que, em geral, os passos se restringem a:

1. substituir valores (em geral escolhidos arbitrariamente) na expressão algébrica de uma função dada;

2. marcar os pontos correspondentes no plano cartesiano;

3. interpolar esses pontos por meio de curvas ou segmentos de retas.

\footnotetext{
1victor@dmm.im.ufrj.br

2luizmc@ime.uerj.br
} 
Este procedimento é, via de regra, a única ferramenta apresentada aos alunos no ensino médio para esboçar gráficos. Verifica-se então que a conexão entre as três principais representações se dá somente da seguinte forma:

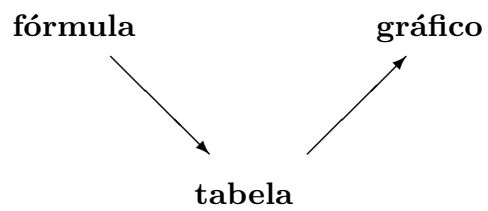

Neste trabalho, propomos uma série de atividades, utilizando computadores ou calculadoras gráficas, planejadas com o objetivo de "completar" o diagrama acima, estimulando o estabelecimento das demais conexões, particularmente a ligação direta entre gráfico e fórmula. No entanto, atividades computacionais de matématica geram outras situações de aprendizagem importantes, como por exemplo as apresentadas em $[1,3,7,8]$. Cabe, então, também discutir as limitações dos programas computacionais atualmente utilizados no ensino de funções e induzir, ainda que intuitivamente, a necessidade do conceito de continuidade.

Este trabalho está dividido em quatro seções, além dessa introdução. Na seção 2. apresentamos uma discussão sobre a aprendizagem de Matemática em ambientes computacionais. Em 3., destacamos quais os conceitos que devem ser evidenciados para completar-se o esquema acima. Exemplos de atividades e suas análises são apresentados na seção 4.. Algumas conclusões preliminares são endereçadas na seção 5 ..

\section{Atitudes Frente a Novas Tecnologias}

Com o aumento da utilização de recursos computacionais no ensino, alguns artigos apontam para deficiências na formação de conceitos matemáticos relacionadas a esse uso.

Monaghan, Sun e Tall [5], por exemplo, comparam os resultados dos cursos iniciais de cálculo em duas escolas inglesas. Na escola $\mathbf{A}$, o curso foi dado nos modelos tradicionais; enquanto que na escola $\mathbf{B}$, baseado em aulas de laboratório, nas quais foi utilizado o aplicativo Derive. Ao final da experiência, foi pedido aos estudantes que explicassem o significado da expressão $\lim _{h \rightarrow 0} \frac{f(x+h)-f(x)}{h}$.

Os autores descrevem os resultados da seguinte forma:

(...) Todos os estudantes na escola $\mathbf{A}$ deram explicações teóricas satisfatórias da expressão, mas nenhum deu exemplos. Entretanto, nenhum do grupo Derive deu explicações teóricas e somente dois estudantes mencionaram as palavras 'gradiente' ou 'diferenciar'. Quatro do grupo Derive deram exemplos. Eles substituíram $f(x)$ por um polinômio e efeturam ou descreveram a seqüência de comandos para calcular o limite.

Esses resultados sugerem que os estudantes da escola B formaram uma imagem de diferenciação mais como um algoritmo do que como um conceito matemático. 
Outros trabalhos revelam as dificuldades, agora de professores, para se lidar com novas tecnologias gráficas no ensino de matemática.

Em sua dissertação de mestrado, Abrahão [1] observou as reações de quatro professores de ensino fundamental e médio diante de gráficos de funções gerados por computadores e calculadoras gráficas. Nas atividades aplicadas, os resultados fornecidos pela máquina eram de forma geral contraditórios com a teoria matemática envolvida, em virtude da inadequação dos programas empregados, ou limitações da janelas gráficas escolhidas para a visualização. Por diversas vezes no decorrer do experimento, os professores hesitaram em considerar o fato de que um computador pode gerar resultados errados ou incompletos. Nestas situações, os resultados chegaram a ser considerados verdadeiros, sem questionamento. A visualização no computador foi assumida como critério absoluto de verdade, mesmo quando claramente contrário à teoria matemática. A autora comenta que:

Quando solicitados a interpretar alguns gráficos não usuais produzidos em computadores, os entrevistados apresentaram algumas dificuldades. Pudemos observar que a compreensão de gráficos gerados por tecnologia gráfica não é imediata. Nem sempre o professor conseguia conciliar seus conhecimentos teóricos com a visualização gráfica.

Mais adiante, na análise dos resultados de uma das atividades, prossegue:

Um dos professores entrevistados, ao ver a janela onde o gráfico da função do $3^{0}$ grau lembrava uma parábola, interpretou esse gráfico parcial como sendo o gráfico global da função cúbica. Nesse momento seus conhecimentos teóricos não foram ativados para fazê-lo perceber que sua interpretação estava incorreta.

Resultados semelhantes são relatados por Belfort e Guimarães [3]. Nesse trabalho, foi observado o comportamento de professores no desenvolvimento de atividades de geometria plana em computadores. Em uma das atividades descritas foi pedido que se encontrasse experimentalmente o retângulo com $40 \mathrm{~m}$ de perímetro e a maior área possível. Com o programa empregado, os professores podiam construir um retângulo com o perímetro dado e variar os lados mantendo o perímetro fixo, observando a variação da área. O retângulo que soluciona o problema é o quadrado de $10 \mathrm{~m}$ de lado e $100 \mathrm{~m}^{2}$ de área. No entanto, devido aos arredondamentos efetuados pelo programa, os resultados gerados pela máquina podiam ser incorretos. Um dos professores cursistas, por exemplo, ao encontrar como solução um retângulo com área $100 \mathrm{~m}^{2}$ e lados $10,03 \mathrm{~m}$ e $9,97 \mathrm{~m}$, teve dificuldades em encontrar uma explicação para esta incoerência. Os autores relatam:

(...) muitos professores aceitavam a resposta dada pelo programa como conclusiva. Em um dos experimentos, fomos chamados por três duplas de professores que, sentados próximos, haviam comparado suas respostas. Todos tinham obtido uma área máxima de $100 \mathrm{~m}^{2}$, mas os valores do lado $A B$ diferiam. Esses professores ficaram em um impasse, e não 
conseguiram decidir qual dos três valores encontrados seria o correto. A exploração do 'erro' do programa levou à necessidade de encontrar uma solução teórica para o problema.

Em outro trabalho, Belfort e Guimarães [2] descrevem uma experiência com o uso de programa de geometria dinâmica na formação continuada de professores, na qual os participantes eram estimulados a formular conjecturas e verificar sua validade. A maioria dos professores teve sucesso em mostrar que suas hipóteses eram verdadeiras ou falsas, por meio de argumentos matemáticos formais ou da apresentação de contra-exemplos, conforme o caso. Mais do que isso, muitos deles reconheceram que esta verificação de hipóteses não pode ser feita por meio da simples visualização na tela do computador, ou, de forma mais geral, através de quaisquer métodos empíricos. Segundo os autores, os professores relatam que os termos "geometria experimental" e "geometria dedutiva" passaram a ser melhor compreendidos. Os autores concluem que:

Finalmente, do ponto de vista de programa de geometria dinâmica em formação de professores, estamos convencidos que é possível utilizá-los não apenas no estágio exploratório do estudo da geometria, mas também como uma ferramenta de apoio para o desenvolvimento da capacidade de justificar resultados e da valorização do raciocínio dedutivo em geometria.

Os exemplos citados sugerem que os "benefícios" ou "malefícios" do uso de novas tecnologias no ensino não são intrínsecos à máquina, mas determinados pelo seu emprego em sala de aula. David Tall [8] afirma que:

O uso de calculadoras e computadores em matemática nem sempre tem tido tanto sucesso quando poderia. Na Inglaterra, o uso de calculadoras com crianças pequenas tem sido desencorajado na esperança de que sua ausência capacitará as crianças a construir relações aritméticas mentais. Talvez esta atitude tenha mais relação com o mal uso da calculadora (para realizara cálculos sem ter que pensar) do que qualquer falha inerente na próprio aparato. Bem usada - para encorajar reflexões em idéias matemáticas - a calculadora pode ser bastante benéfica (...)

Verificamos ainda que não se estabelece de forma simples e natural a ponte entre as representações computacionais dos objetos matemáticos e as respectivas formulações teóricas. Entretanto, nos alinhamos com os autores citados na opinião de que é justamente na construção desta ponte - particularmente com a ocorrência de contradições - que as novas tecnologias podem se colocar como um recurso didático rico para o ensino de matemática. 


\section{Algoritmos Computacionais e Estratégias Humanas}

Na concepção das atividades aqui propostas, destacamos inicialmente a comparação entre propriedades matemáticas de duas naturezas distintas:

- quantitativas: dizem respeito aos valores de uma função em um subconjunto finito de pontos do domínio;

- qualitativas: envolvem os valores de uma função no domínio globalmente, num intervalo, ou, de forma geral, num subconjunto infinito do domínio.

Por exemplo, a existência de descontinuidades numa dada função $f: \mathbb{R} \rightarrow \mathbb{R}$ é uma propriedade qualitativa, uma vez que sua determinação exige a comparação dos valores de $f$ numa vizinhança dos pontos em questão.

O procedimento comumente empregado por alunos para esboçar gráficos, conforme o descrito anteriormente, lança mão somente de propriedades quantitativas da função, isto é, de seus valores num conjunto finito de pontos do domínio. Da mesma forma, os algoritmos computacionais para gerar gráficos se baseiam na determinação de uma quantidade finita de valores. A diferença aqui é clara: sendo a capacidade de cálculos da máquina imensamente maior que a humana, esses pontos ficam suficientemente próximos para que se crie a ilusão de se estar visualizando uma curva na tela.

Em contrapartida, grande parte das propriedades qualitativas que um indivíduo pode usar para esboçar gráficos não são acessíveis aos programas atuais. Essa limitação pode dar origem a situações onde a representação computacional não corresponde ao modelo matemático.

Observemos o exemplo a seguir. Na figura 1, vemos os gráficos das funções $f(x)=\frac{1}{(x-1)}$ e $g(x)=\frac{1}{(x-1)^{2}}$, respectivamente, gerados pelo aplicativo Maple.
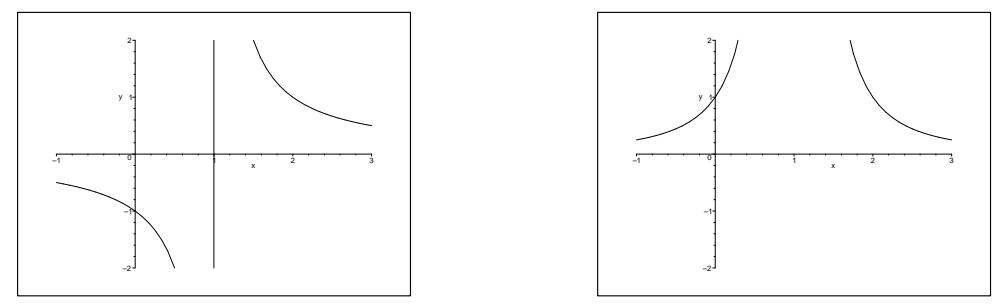

Figura 1: As curvas $f(x)=\frac{1}{(x-1)}$ e $g(x)=\frac{1}{(x-1)^{2}}$, com a ocorrência de uma assíntota aparente.

Ambas as funções possuem assíntotas verticais em $x=1$, no entanto esta reta só aparece nos gráfico de $f$. Na verdade, o programa não reconhece a ocorrência das assíntotas para nenhuma das duas curvas. A reta que aparece no gráfico de $f$ é gerada pela interpolação indevida de um ponto a esquerda com um a direita 
de $x=1$, isto é, o programa trata esta reta como uma parte do gráfico da função $f$. O mesmo não ocorre na função $g$ por que os dois limites laterais em $x=1$ são positivos.

Nesse exemplo, o gráfico gerado pelo computador é incorreto, pois não é utilizada a informação qualitativa de que a função $f$ possui uma descontinuidade em $x=1$.

Na realidade, o Maple dispõe de uma opção que permite ao usuário informar que o programa deve verificar a existência de descontinuidades antes de traçar o gráfico, o que, neste caso, evitaria a ocorrência do erro. No entanto, esta verificação é feita por meio da análise da expressão algébrica da função.

Tanto por humanos quanto por computadores, as propriedades qualitativas de uma função - como é o caso da continuidade - não podem ser verificadas de forma direta, ou seja, por meio, da determinação efetiva de infinitos valores. Há, pelo menos, duas estratégias possíveis para se tratar esses casos. A primeira, algébrica, analisa a função por meio de sua fórmula. A outra, lógico-formal, utiliza diretamente as definições matemáticas.

Os programas do tipo CAS (Computer Algebra Systems), como o Maple, podem efetuar, pelo menos até certo ponto, análises simbólicas de expressões algébricas. Entretanto, a combinação da análise algébrica com a argumentação lógico-formal ainda é uma estratégia exclusivamente humana para lidar com propriedades qualitativas.

\section{Exemplos de Atividades Propostas}

Nas atividades a seguir, observamos o gráfico de uma mesma função em diferentes janelas gráficas, apresentando aspectos distintos. Estes aspectos são determinados fundamentalmente por dois fatores: a relação entre as ordens de grandeza utilizadas nos eixos coordenados e os erros gerados e as limitações dos algoritmos computacionais quantitativos. Cada janela gráfica observada fornece um tipo específico de informação sobre a função em questão. A compreensão do comportamento global da função se desenvolverá a partir da conexão entre gráfico e fórmula, em conjunto com a correlação entre estas diferentes informações, e da crítica aos erros e limitações do programa. Neste processo, são evidenciadas propriedades qualitativas, através da conexão direta entre gráfico e fórmula.

Exemplo 1: Na figura 2, vemos o gráfico da função $h(x)=\frac{1}{x^{4}+1000}$, em três janelas gráficas diferentes, respectivamente: $x \in[-1,1]$ e $y \in[0,1] ; x \in[-1,1] \mathrm{e}$ $y \in[0,0.002] ; x \in[-10,10]$ e $y \in[0,0.002]$.

Como na primeira janela, o intervalo escolhido para $y$ é grande demais para os valores de $h$, o gráfico é representado no computador como uma reta horizontal muito próxima do eixo $x$. A seguir, diminuimos o intervalo de $y$, mas como $x$ continua pequeno, os valores de $x^{4}+1000$ ficam muito próximos de 0.001 , logo o gráfico é representado como uma reta horizontal em $y=0.001$. Na terceira janela, aumentamos o intervalo de $y$, obtendo uma boa visualização das regiões de crescimento e decrescimento de $h$. 

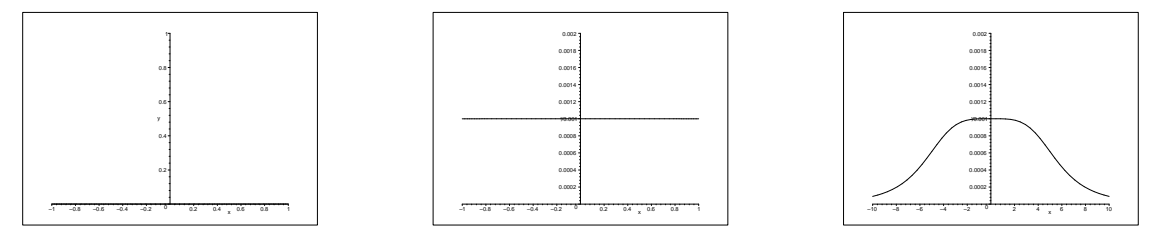

Figura 2: $h(x)=\frac{1}{x^{4}+1000}$

Exemplo 2: A figura 3 representa o gráfico do polinômio $q(x)=\left(x^{2}-2\right)(5 x-7)$, respectivamente nas janelas: $x \in[-3,3]$ e $y \in[-20,20] ; x \in[-3,3]$ e $y \in$ $[-0.5,0.5] ; x \in[1.3,1.5]$ e $y \in[-0.005,0.005]$.
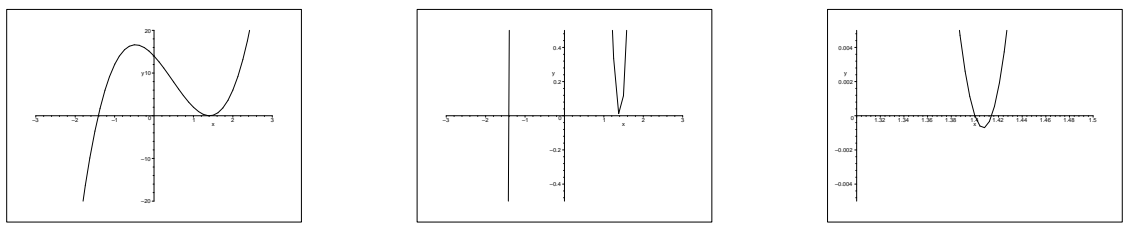

Figura 3: $q(x)=\left(x^{2}-2\right)(5 x-7)$

As raízes positivas de $q, x_{1}=\sqrt{2}$ e $x_{2}=\frac{7}{5}$, diferem apenas na casa dos centésimos, portanto ficam indistingüíveis na primeira janela gráfica. Na segunda, o passo de interpolação faz com que o gráfico traçado sequer toque o eixo horizontal próximo a esses dois pontos. Na última, ao aproximarmos a janela de $x_{1}$ e $x_{2}$, conseguimos distingüir as duas raízes.

Através deste exemplo, podem ser evidenciadas as relações entre a fatoração de um polinômio e o comportamento de seu gráfico, no que diz respeito à multiplicidade das raízes.

Exemplo 3: Em 4, temos o gráfico de $s(x)=\operatorname{sen}(1000 x)+1000 \operatorname{sen}(x)$, para $x \in[-2 \pi, 2 \pi]$ e para $x \in\left[\frac{\pi}{2}-0.01, \frac{\pi}{2}+0.01\right]$.
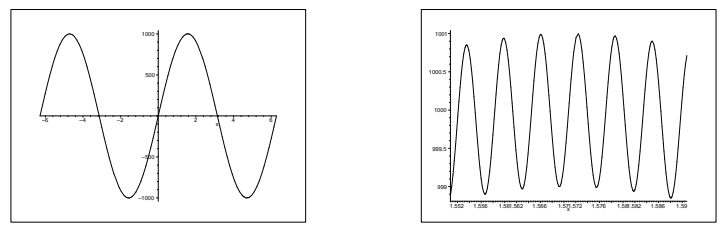

Figura 4: $s(x)=\operatorname{sen}(1000 x)+1000 \operatorname{sen}(x)$

Como a amplitude da parcela sen $(1000 x)$ é muito menor que a de 1000 sen $(x)$, na janela da esquerda o gráfico se assemelha muito ao de $y=1000 \operatorname{sen}(x)$. Na segunda janela, o contrário ocorre. Como os valores de $x$ são agora muito menores, o gráfico adquire um aspecto semelhante ao de $y=\operatorname{sen}(1000 x)$. 
Neste exemplo, são exploradas relações entre funções periódicas com freqüências e amplitudes de ordem de grandeza muito diferentes.

Exemplo 4: A figura 5, mostra o gráfico de $u(x)=\operatorname{sen}(\ln x)$, para $x \in] 0,10]$, para $x \in] 0,1]$ e para $x \in] 0,0.1]$.
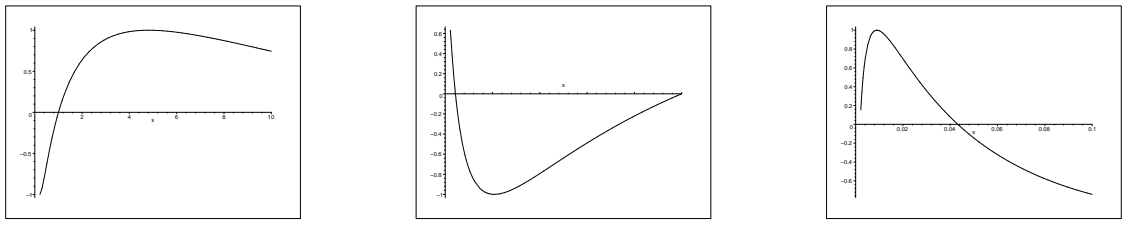

Figura 5: $u(x)=\operatorname{sen}(\ln x)$

As raízes de $u$ são os números reais $x_{k}=\mathrm{e}^{k \pi}$, com $k \in \mathbb{Z}$. Estas formam portanto um conjunto que se acumula em 0 . Nas figuras acima, entre os trechos do gráfico desenhados pelo programa e o eixo vertical, a curva oscila infinitas vezes entre -1 e 1 . Como as raízes estão em progressão geométrica, cada uma dessas oscilações se torna imperceptível em relação à anterior. Isto faz com que o gráfico adquira aspectos radicalmente diferentes em cada janela, o que não ocorre, por exemplo, com a funçãosen $(1 / x)$. Desta forma, nenhuma delas pode representar o comportamento de $u$ de forma global. A compreensão de tal comportamento, portanto, só pode se dar por meio da análise qualitativa da função, abstraindo a representação computacional.

\section{Considerações Finais}

Não se estabelece de forma simples e natural a relação entre as representações computacionais dos objetos matemáticos e suas formulações teóricas. Entretanto mostramos vários exemplos em que é justamente na construção desta relação que computadores e calculadoras gráficas podem se colocar como um recurso didático importante para o ensino de matemática.

Tall [9] define um organizador genérico, como sendo um ambiente que possibilita o estudante manipular exemplos e (se possível) contra-exemplos de um conceito matemático específico ou de um sistema de conceitos relacionados. Em [7], o autor afirma que:

Eu espero de todos os organizadores genéricos que estes 'contenham as sementes da sua própria destruição', no sentido em que eles devem ser suficientemente sofisticados para mostrar as limitações de seus processos de modelagem e a necessidade de abordagens teóricas mais amplas.

Exemplos como os aqui apresentados podem ser usados para mostrar como algoritmos computacionais quantitativos podem gerar resultados conflitantes com a 
teoria matemática associada e, portanto, como estes devem ser analisados criticamente. A partir daí, poderá ser motivado o estudo de propriedades qualitativas, em particular por meio da conexão direta entre gráfico e fórmula.

Abstract. We address and analyze some computational activities for the teaching of real functions designed for secondary teachers and undergraduate students. We aim to stimulate the connections between three main function representations (analytical, graphical and numerical), critically analyzing unexpected results generated by computers.

\section{Referências}

[1] A.M.C. Abrahão, "O Comportamento de Professores Frente a Alguns Gráficos de Funções $f: \mathbb{R} \rightarrow \mathbb{R}$ Obtidos com Novas Tecnologias", Dissertação de Mestrado, Departamento de Matemática, PUCRJ, Rio de Janeiro, RJ, 1998.

[2] E. Belfort e L.C. Guimarães, Uma experiência com software educativo na formação continuada de professores de matemática, em "Anais do VI Encontro Nacional de Educação Matemática", Vol. II, pp. 376-379, SBEM, 1998.

[3] E. Belfort, L.C. Guimarães e R. Barbastefano, Geometria dinâmica e demonstrações na formação continuada de professores, em "Anais do Cabri World 99", Vol. eletro, Cabri World, 1999.

[4] V. Giraldo e L.M. Carvalho, Funções e novas tecnologias: algumas perguntas, em "Anais do III Seminário: A Pesquisa em Educação Matemática no Rio de Janeiro", Vol. 1, pp. 24-29, SBEM/RJ, 2000.

[5] J.D. Monaghan, S. Sun e D. Tall, Construction of the limit concept with a computer algebra system, em "Proceedings of the International conference of the International Group for Psycology of Mathematics Education", (J.P. da Ponte e J.F. Matos, eds.), Vol 3, pp. 279-286, ICME, 1993.

[6] A. Sierpinska, On understanding the notion of function, em "MAA Notes and Report Series" (G. Harel e E. Dubinsky, eds.), Vol. 4, pp. 25-58. 1992.

[7] D.O. Tall, Biological brain, mathematical mind \& computational computers, em "ATCM Conference", pp. 1-18, ATCM, 2000.

[8] D.O. Tall, Cognitive development in advanced mathematics using technology, Mathematics Education Research Journal, 12 (2000), 210-230.

[9] D.O. Tall, Concept images, generic organizers, computers \& curriculum change For the Learning of Mathematics, 9 (1989), 37-42. 
